\title{
Learning and survival memory undergoing a permanent bilateral carotid ligation in rats ${ }^{1}$
}

\author{
Maria Cecília Santos Cavalcanti MeloI, Diego Gadelha"I, Guilherme Veras Mascena ${ }^{\mathrm{III}}$, Thárcia Kiara Beserra Oliveira ${ }^{\mathrm{IV}}$, Carlos \\ Teixeira Brandt ${ }^{\mathrm{V}}$ \\ IMaster, Assistant Professor, Department of Ophthalmology, FCM, Campina Grande-PB, Brazil. Acquisition, interpretation of data, manuscript writing. \\ IIFellow PhD degree, Postgraduate Program in Surgery, Health Sciences Center, Federal University of Pernambuco (UFPE). Associate Professor, \\ Department of Ophthalmology, FCM, Campina Grande-PB, Brazil. Manuscript writing, critical revision. \\ IIIAssistant Professor, Department of Cardiology, FCM, Campina Grande-PB, Brazil. Interpretation of data, manuscript writing. \\ ${ }^{\mathrm{IV}}$ Veterinarian, Animal Colony, FCM, Campina Grande-PB, Brazil. Acquisition and interpretation of data. \\ ${ }^{v} \mathrm{PhD}$, Head Professor, Scientific Methodology and Pediatric Surgery, UFPE, Pernambuco, Brazil. Scientific and intellectual content of the study, \\ interpretation of data, critical revision.
}

\section{ABSTRACT}

PURPOSE: To evaluate the effect of cerebral hypoxia-ischemia on memory and learning survival of rats submitted to permanent bilateral carotid ligation (PBCL).

METHODS: Twenty-four survivors of PBCL were evaluated after 30 days with regard to memory and learning using a water survival maze. Twenty-three healthy rats were used as control group. The results were expressed by their means and standard error of the mean (SEM). $\mathrm{p}<0.05$ was used for rejecting the null hypothesis. The study was approved by the Ethics Committee for animal investigation.

RESULTS: The mortality rate for the surgery was $44.4 \%$. The latency time to find the survival platform was higher in rats that underwent PBCL (Normal: $10.24 \pm 1.85 \mathrm{~s}-$ Study: $25.30 \pm 4.69 \mathrm{~s}-$ Mann-Whitney $\mathrm{p}=0.0388$ ). Additionally, the type of swimming and the spatial stability of the studied rats on the survival platform were compromised in these animals.

CONCLUSION: The permanent bilateral carotid ligation induces change in the learning and survival memory.

Key words: Hypoxia-Ischemia, Brain. Carotid Arteries. Memory Disorders. Rats. 


\section{Introduction}

Permanent bilateral occlusion of the common carotid arteries (PBOCA) is a model of chronic cerebral hypo perfusion leading to cognitive impairment. It also affects the retina and optic nerve, thus providing a useful model for investigating the mechanisms of neuron losses and therapy for degeneration associated with vascular defects ${ }^{1,2}$.

PBOCA in rats decreases the cerebral blood flow to about $50 \%$ normal, but gives no significant changes in the energy state of the central nervous tissue ${ }^{3}$.

The main findings after PBOCA are in the brain cortex and hippocampus ${ }^{4}$.

PBOCA impairs the working memory, however the damage to the brain tissues and the visual system have been disputed $^{5}$. Furthermore, the clinical implication that a reduction in blood flow may be an important factor that causes or exacerbates cognitive decline in dementias of human beings ${ }^{6}$ gives a special interest in the outcome of the present study. In rat model of the long-lasting reduction of cerebral blood flow, produced by PBOCA, combined with ageing can result in the enhanced cognitive impairment of aged individuals ${ }^{7}$.

Other perspective of this investigation is to interfere in the natural history of persistent encephalic hypoxia using environmental enrichment or substances as a way to produce neuroprotective effect against the cognitive impairment by chronic cerebral hypo perfusion due to $\mathrm{PBOCA}^{8-10}$. The experimental model can also allow observing different chronic non communicant chronic diseases related to ageing such as: degenerative macular disease associated to ageing, and to injecting intraocular substances with the goal of protecting the retina from this degeneration during the regional hypoxemia ${ }^{11}$.

The purpose of this study was to investigate the influence of cerebral hypoperfusion on learning-memory function in rats, in which the PBOCA reduced the cerebral blood flow, and examined its cognition and survival behavior using a water maze, adapted from the Morris water maze ${ }^{12}$.

\section{Methods}

The research project was approved by the Research Ethics Committee of FCM- Campina Grande-PB, Brazil.

Twenty-four adult Wistar rats (Rattus norvegicus) from the animal colony were used for this study. They were housed in polypropylene cages, four animals per cage, under standard light/ dark conditions (lights on 7:00, off 19:00) with food pellets and water ad libitum.

Chronic cerebral hypoperfusion was induced by PBOCA $^{1,2,5,6}$. The animals were anesthetized with ketamine hydrochloride (50 mg/kg ip) and xilasine (10 mg/kg ip). The common carotid arteries were exposed via a ventral midline incision, carefully separated from their sheaths and vagus nerves, and permanently doubly ligated with $5 / 0$ silk suture approximately 8 to $10 \mathrm{~mm}$ below the origin of the external carotid artery.

After four weeks the rats were tested for memory and learning, using a survival water maze task (SWMT). The apparatus consisted of a rectangular water tank measuring $120 \mathrm{~cm}$ in length by $80 \mathrm{~cm}$ wide and $60 \mathrm{~cm}$ in height. To make the water opaque, 1 $\mathrm{kg}$ of powdered milk was added, and water temperature was kept at $23 \pm 1{ }^{\circ} \mathrm{C}$. A translucent acrylic platform $(10 \mathrm{~cm}$ in diameter $)$ was located in the one end (north) of the maze during training. The top of the platform was approximately $1.5 \mathrm{~cm}$ below the surface of water. Spatial training of the platform in the water maze was performed for five consecutive days. Each rat received two trials per day for five days with the inter trial interval of twelve hours.

The starting position (south end) for each trial was randomly chosen and counterbalanced across all experimental groups.

The rats were gently placed into the water, facing the middle position of the maze south side wall. Swimming paths of the rats were monitored by a video camera. For each training trial, the latency to escape onto the platform and the path length were recorded. All data were fed into a computer program for posterior independent analysis.

The rats were given a maximum of 60 s to find the platform. If they failed to find it within $60 \mathrm{~s}$, the training was terminated and a maximum score of $60 \mathrm{~s}$ was assigned. The rats were then guided to the platform by hand, and it was allowed to stay on it for $10 \mathrm{~s}$ before removed from the water.

The physical findings, the way of swimming, balance instability and weakness of the limb muscles were also recorded and analyzed. Both the training process and the sixth day evaluations were performed in a randomized fashion in order to eliminate any bias in the analysis.

The quantitative variables were expressed by their mean and standard error of the mean (SEM) and the qualitative by their frequencies. $\mathrm{p}<0.05$ was used for rejecting the null hypothesis.

\section{Results}

Operative mortality was $44.4 \%$. Latency times in the control group varied from 2.28 to $36.00 \mathrm{~s}(10.24 \mathrm{~s} \pm 1.85 \mathrm{~s}-$ Mean 
\pm SEM). In the study group latency times varied from 3.00 to $60.00 \mathrm{~s}$; without counting overtimes of $60 \mathrm{~s}$, which occurs in five rats; for the sake of the animals they were removed from the water maze (25.26s $\pm 4.69 \mathrm{~s}-$ Mean $\pm \mathrm{SEM}$ ) (Figure 1).

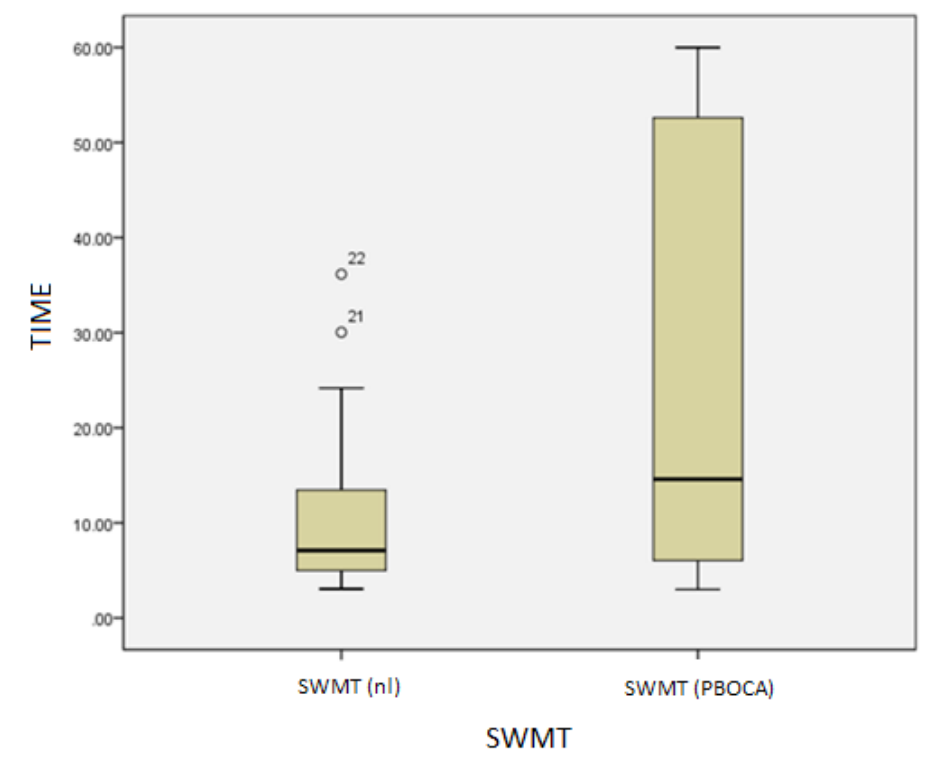

FIGURE 1 - Box plot with latency times (normal and study groups).

In the control group all, but one had a straight swimming towards to the survival platform; however one that spent 36 s for landing in the survival platform performed two spins across the water maze and was a kind of disoriented or not interested in landing in the platform. Three other rats, although had a straight swimming toward the survival platform performed two spins around it before safe and comfortable landing.

The rats that underwent PBOCA presented a physical finding common to the majority $(80 \%)$ of animals: they presented eyelid ptosis in one or both eyes. Two rats, apart from bilateral ptosis, developed eye infected hemorrhagic secretion (conjunctivitis); one of them suffered bullying from two others in the cage and died in the $5^{\text {th }}$ week of clinical observation

At the beginning of the five days training for the tests, the studied rats have decreased strength in the posterior legs; which improved after the training period.

\section{Discussion}

PBOCA has been used as a model of chronic cerebral hypoperfusion. The main findings include histopathological damage of the encephalon and impaired spatial learning function ${ }^{2,13}$. This cognitive impairment may be related to progressive loss of hippocampal pyramidal neurons, an association often observed in human aging and dementia states ${ }^{13}$. In this model, there is an abrupt reduction of whole brain blood flow, ranging from approximately $35-45 \%$ in the cortex area to $60 \%$ in the hippocampus ${ }^{3,4,14,15}$. This hypoperfusion is believed to sustain a chronic state of moderate hypoglycemia, a pathophysiological condition closely resembling that of reduced cerebral blood flow present in human aging and dementia ${ }^{13}$.

Adaptations of the protocol have been explored in order to refine the experimental model by avoiding the abrupt reduction of cerebral blood flow. A modified procedure permitting the gradual establishment of cerebral hypoperfusion by PBOCA. It has been proposed, with a 1-week interval between the occlusion of both common carotid arteries ${ }^{14}$. The purpose of these modifications is to decrease the very high mortality of the surgical approach when the carotids are simultaneously occluded as it happens in the present investigation, in which the overall mortality was within the range of other series ${ }^{15}$. On the other hand, aiming to produce a brain damage close related to the human being it seems that is better do perform PBOCA, rather than a 1-week interval carotid unilateral occlusion, because although it is associated with less mortality rate in the later, the rats have a better hemodynamic adaptations diminishing the repercussions in the cognitive events. Greater cognitive disruptions in rats, as it happens with PBOCA, give a better experimental model to study the best way to positively interfere with the natural history of chronic encephalon ischemia associated with dementia as it occurs in human beings; especially when it is associated with cerebral stroke.

In the present study it was used an adaptation of the Morris water maze procedure ${ }^{12}$ without changing the main purpose of the test, which is to assess the spatial and learning survival memory. Instead of a circular maze it was used a rectangular one. The visual clues were of gray color suitable for the rat vision.

The rats from both groups underwent the same training protocol and visual clues. All the swimming pathway were video recorded and after editing the results they were computed analyzed regarding latency time, length of water path and swimming quality. A veterinary registered any deficiency in rat movements, including muscle weakness and stability on the survival platform. The choice for testing the rats at four weeks after PBOCA was based on the assumption that hypoxia inducible factor 1 (HIF-1 $\alpha$ ) levels reach the peak at the $2^{\text {nd }}$ week after surgery in the hippocampus, while apoptotic neurons were maximal at the $4^{\text {th }}$ week in the this region, especially in the cornu ammonis 1 (CA1) region ${ }^{3}$. Thus, one can speculate that the artery blood flow has already been regained to the brain through the collaterals from the posterior branch coming from the vertebral arteries. In this way, the brain damage has already been stabilized. The almost normal swimming pattern and 
close to normal latency time in some rats of the study group gives support to this hypothesis.

Some of the normal control rats (11 out of $23-47.8 \%$ ), after training, performed a straight way of path towards to the survival platform in three to five seconds, proving to have a normal spatial memory in the water maze. The normal rat that spent about 36 seconds to land in the survival platform did seems enjoying swimming without any fear; representing a point outside the normal time course of the test. Thus, the authors believed that the maze shape made no difference in water survival test analysis, when compared with the original proposed by Morris ${ }^{12}$.

The significant increase in the latency time, the way of swimming, and the use of the tail as a propeller, and the instability of the studied rats on the survival platform do indicate encephalic damage after PBOCA. On the other hand, it was expected a greater brain function alterations. One can speculate that the return of the brain arterial blood through the posterior tributaries coming from the vertebral arteries have attenuated the further brain damage. The decrease of apoptosis in the hippocampus after four postoperative follow-up in rats that underwent PBOCA gives further support to this hypothesis ${ }^{4}$.

The importance of the present study, apart from testing one of the cognitive function (spatial memory for survival purpose), and to validate the animal model for brain hypoxemia is to maintain in the animal house a group of brain ageing rats that can be used for intervention in the natural history of chronic encephalic hypoxemic animals, in order to further investigate ways of preventing precocious dementia in human beings. As well to better manage the increasing non communicant ageing disease, as expectative of long life is predicted for human society. For further investigations one can test several substances, particularly natural functional foods and active way of life to improve neurocognitive functions in order to produce better outcome that can help human being medicine for future approach for ageing and dementia development.

\section{Conclusions}

The PBOCA induces change in the learning and survival memory increasing the latency time for survival test in the water maze. The mortality rate to produce the rat model for chronic brain hypoxemia is inside the range of other series.

\section{References}

1. Yamamoto H, Schmidt-Kastner R, Hamasaki DI, Yamamoto H,

Parel JM. Complex neurodegeneration in retina following moderate ischemia induced by bilateral common carotid artery occlusion in Wistar rats. Exp Eye Res. 2006;82:767-79.

2. Tsuchiya M, Sako K, Yura S, Yonemasu Y. Cerebral blood flow and histopathological changes following permanent bilateral carotid artery ligation in Wistar rats. Exp Brain Res. 1992;89(1):87-92.

3. Eklöf B, Siesjö BK. Cerebral blood flow in ischemia caused by carotid artery ligation in the rat. Acta Physiol Scand. 1973;87(1):6977.

4. Yu CH, Moon CT, Sur JH, Chun YII, Choi WH, Yhee JY. Serial expression of hypoxia inducible factor- 1 alfa and neuronal apoptosis in hippocampus of rats with chronic ischemic brain. J Korean Neurosurg Soc. 2011;50:481-5.

5. Ohta $H$, Nishikawa $H$, Kimura $H$, Anayama $H$, Miyamoto $M$. Chronic cerebral hypoperfusion by permanent internal carotid ligation produces learning impairment without brain damage in rats. Neuroscience. 1997;79:1039-50.

6. Scherr M, Trinka E, Mc Coy M, Krenn Y, Staffen W, Kirschner M, Bergmann HJ, Mutzenbach JS. Cerebral hypoperfusion during carotid artery stenosis can lead to cognitive deficits that may be independent of white matter lesion load. Curr Neurovasc Res. 2012;9(3):193-9

7. Sopala M, Danysz W. Chronic cerebral hypoperfusion in the rat enhances age-related deficits in spatial memory. J Neural Transm. 2001;108(12):1445-56.

8. Cechetti F, Worm PV, Lovatel G, Moysés F, Siqueira IR, Netto CA. Environmental enrichment prevents behavioral deficits and oxidative stress caused by chronic cerebral hypoperfusion in the rat. Life Sci. 2012;91(1-2):29-36.

9. Goo MJ, Choi SM, Kim SH, Ahn BO. Protective effects of acetyl-Lcarnitine on neurodegenarative changes in chronic cerebral ischemia models and learning-memory impairment in aged rats. Arch Pharm Res. 2012;35(1):145-54.

10. Shang YZ, Miao H, Cheng JJ, Qi JM. Effects of amelioration of total flavonoids from stems and leaves of Scutellaria baicalensis Georgi on cognitive deficits, neuronal damage and free radicals disorder induced by cerebral ischemia in rats. Biol Pharm Bull. 2006;29(4):805-10.

11. Ikram MK, Mitchell P, Klein R, Sharrett AR, Couper DJ, Wong TY. Age-related macular degeneration and long-term risk of stroke subtypes. Stroke. 2012;43(6):1681-3.

12. Morris R. Developments of a water-maze procedure for studying spatial learning in the rat. J Neurosci Methods. 1984;11(1):47-60.

13. Farkas E, Luiten PG, Bari F. Permanent bilateral common carotid artery occlusion in the rat: a model for chronic cerebral hypoperfusion-related neurodegenerative diseases. Brain Res Rev. 2007;54:162-80.

14. Cechetti F, Worm PV, Pereira LO, Siqueira IR, A Netto C. The modified $2 \mathrm{VO}$ ischemia protocol causes cognitive impairment similar to that induced by the standard method, but with a better survival rate. Braz J Med Biol Res. 2010;43(12):1178-83.

15. Farkas E, Timmer NM, Domoki F, Mihaly A, Luiten PG, Bari F. Post-ischemic administration of diazoxide attenuates long-term microglial activation in the rat brain after permanent carotid artery occlusion. Neurosci Lett. 2005;387:168-72

\section{Correspondence:}

Carlos Teixeira Brandt

Avenida Boa Viagem, 5030/1302

51011-000 Recife - PE Brasil

Tel.: (55 81)3342-0830

carlosbrandt@bol.com.br

${ }^{1}$ Research performed at Experimental Research Unit, Medical Sciences, Faculty of Campina Grande (FCM), Paraiba, Brazil.
Received: September 10, 2012

Review: November 12, 2012

Accepted: December 11, 2012

Conflict of interest: none Financial source: Medical Sciences, Faculty of Campina Grande 